\title{
Estudo comparativo de técnicas numéricas de inversão para obtenção de distribuição de tamanho de gotas em emulsões
}

\author{
C. F. B. da $\operatorname{SILVA}^{1}$, C. ASSENHAIMER ${ }^{1}$ e R. GUARDANI ${ }^{1}$ \\ ${ }^{1}$ Universidade de São Paulo, Escola Politécnica, Departamento de Engenharia Química \\ E-mail para contato: carlosfbueno@gmail.com
}

RESUMO - Este trabalho tem como objetivo a implementação e comparação de técnicas para resolução de problemas de inversão, desenvolvendo algoritmos que forneçam distribuições de tamanho de partículas em dispersões a partir de dados de espectroscopia UV-Vis.

\section{INTRODUÇÃO}

O desenvolvimento de algoritmos computacionais para a obtenção de distribuição de tamanhos de partículas em dispersões e que utilizam dados espectroscópicos em tempo real e "in-line" a partir de sensores, permitirá uma variedade de aplicações, que vão desde o acompanhamento de processos de polimerização, ao tratamento de efluentes e sensoriamento atmosférico.

O presente estudo visa o desenvolvimento de algoritmos para a obtenção de distribuições de tamanhos de partículas a partir de dados de espectroscopia UV-Vis. Os casos que serão apresentados a seguir terão como foco as emulsões. O problema matemático a ser resolvido pode ser ilustrado na expressão a seguir:

$$
\tau\left(\lambda_{0}\right)=\frac{\pi}{4} \int_{0}^{\infty} Q_{\text {ext }}\left(\lambda_{0}, D\right) D^{2} f(D) d D
$$

$\mathrm{Na}$ expressão, os valores de turbidez $(\tau)$ e respectivos comprimentos de onda $\left(\lambda_{0}\right)$ são conhecidos. O termo $Q_{\text {ext }}$ corresponde a eficiência de extinção e também pode ser determinado. A função de distribuição de gotas $f(D)$ é o termo desconhecido da expressão. Seinfeld e Pandis (2006) explicam sucintamente a relação entre os espectros de turbidez e as distribuições de tamanho de partículas mostrada da equação acima. Discretizando a Equação 1, obtém-se:

$$
\tau=A f
$$

O termo A corresponde a uma matriz que contém informações ópticas, cuja inversa é difícil de ser obtida por ser uma matriz quase-singular. Faz-se necessário, neste caso, a utilização de uma técnica de inversão apropriada. Os princípios básicos dos problemas de inversão pode ser encontrados em Twomey (1977). 


\section{ALGORITMOS TESTADOS}

\subsection{Non-Negative Least Squares (NNLS)}

A técnica NNLS é uma das alternativas para solucionar o problema de inversão da matriz A. Trata-se de uma versão da técnica de minimização quadrática, com a inserção de uma restrição de não-negatividade nos componentes da função de distribuição final. Matematicamente, tem-se:

$$
\min _{f}\|A f-\tau\|_{2} \text { tal que } f \geq 0
$$

As propriedades ópticas necessárias para a construção da matriz $A$ estão baseadas na teoria de Mie e foram calculadas a partir dos scripts elaborados por Mätzler (2002 a, b). Foi utilizada a rotina lsqnonneg contida na biblioteca de funções do MATLAB ${ }^{\circledR}$ para a resolução do problema de inversão desta matriz. O detalhamento da técnica é apresentado por Lawson e Hanson (1974).

\subsection{Philips-Twomey Regularization (PTR)}

A técnica PTR também tem como função a resolução de problemas de inversão de matrizes quase-singulares. Ela propõe que a Equação 2 seja reescrita, inserindo-se alguns parâmetros auxiliares. A principal característica dessa técnica está na suavização da resposta final.

$$
f=\left(A^{T} A+\gamma H\right)^{-1} A^{T} \tau
$$

A construção da matriz $A$ é demonstrada por Elicabe e Garcia-rubio (1990). Os cálculos das propriedades ópticas realizados pelos algoritmos estão baseados no modelo de Mie e os scripts auxiliares são detalhados por Mätzler (2002 a ,b).

O termo $\gamma$ é chamado de parâmetro de regularização e seu cálculo é feito utilizando a técnica Generalized Cross Validation (GCV). Essa técnica, assim como os valores da matriz $H$ (matriz de suavização), são demonstrados por Elicabe e Garcia-rubio (1990).

\section{MÉTODOS DE GERAÇÃO E COMPARAÇÃO DE RESULTADOS}

Nesta etapa do trabalho, apenas dados artificiais foram utilizados. Ou seja, foram utilizados scripts em MATLAB ${ }^{\circledR}$ para criação de distribuições de tamanho de gotas e espectros de extinção artificiais, o que elimina o trabalho de realizar procedimentos experimentais para obtenção de dados. Etapas futuras do trabalho visam testes com dados reais.

O procedimento consiste de três etapas:

Etapa 1. Construção de distribuições de tamanho de gotas monomodais - que serão denominadas como distribuições originais. 
Nesta etapa, foi utilizada a rotina lognpdf do MATLAB $^{\circledR}$ para criar as distribuições:

$$
Y=\operatorname{lognpdf}(X, \mu, \sigma)
$$

Os valores da Tabela 1 foram utilizados como parâmetros de geração das distribuições. Ao todo, foram criadas 12 distribuições através da combinação dos parâmetros $D g$ e $\sigma$.

Tabela 1 - Parâmetros de geração

\begin{tabular}{|c|c|}
\hline $\mathrm{Dg}(\mathrm{nm})$ & $\sigma$ \\
\hline 500 & 0,1 \\
\hline 1000 & 0,2 \\
\hline 1500 & 0,3 \\
\hline 2000 & \\
\hline
\end{tabular}

Onde $\mu=\ln (D g)$.

$\mathrm{O}$ vetor $\mathrm{X}$ corresponde as classes de tamanho de partícula. Abaixo é mostrado como foram definidos o valor mínimo, o tamanho de passo e o valor máximo deste vetor:

$$
X=\left(0,1 D g: \frac{2 D g-0,1 D g}{100}: 2 D g\right)
$$

Etapa 2. Construção de espectros de extinção a partir dos dados das distribuições monomodais originais.

Bohren e Huffman (1983) detalham todas as premissas do modelo de Mie, que foi o modelo utilizado para os cálculos ópticos e construção dos espectros.

Etapa 3. Recriação das distribuições de tamanho de gotas a partir dos algoritmos de inversão utilizando os espectros da etapa anterior.

Utilizou-se os algoritmos citados anteriormente, NNLS e PTR, para a solução da Equação 2 e consequente criação de distribuições de tamanho de partículas através dos espectros de turbidez criados na etapa 2.

De posse das distribuições original (etapa 1) e recriada (etapa 3), deve-se estabelecer critérios comparativos para avaliar a eficiência das técnicas utilizadas. Os critérios escolhidos foram os mais tradicionais, que consistem na comparação do valor médio e do desvio-padrão entre a distribuição original e a recriada.

\section{RESULTADOS E DISCUSSÃO}

Os dados mostrados a seguir foram simulados utilizando informações ópticas (expressões para 
índice de refração) relativas a uma emulsão de glicerol em água.

A seguir, será apresentado um comparativo entre as distribuições original e recriadas, para um dado diâmetro médio e desvio-padrão da distribuição original.

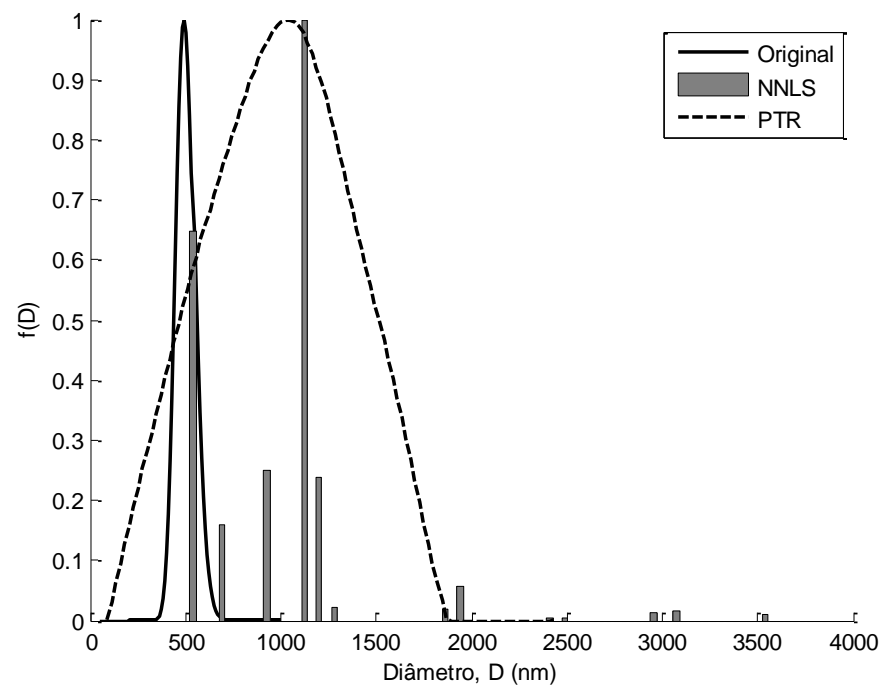

Figura 1 - Diâmetro médio $=502,51 \mathrm{~nm}$ e DP $=50,38$

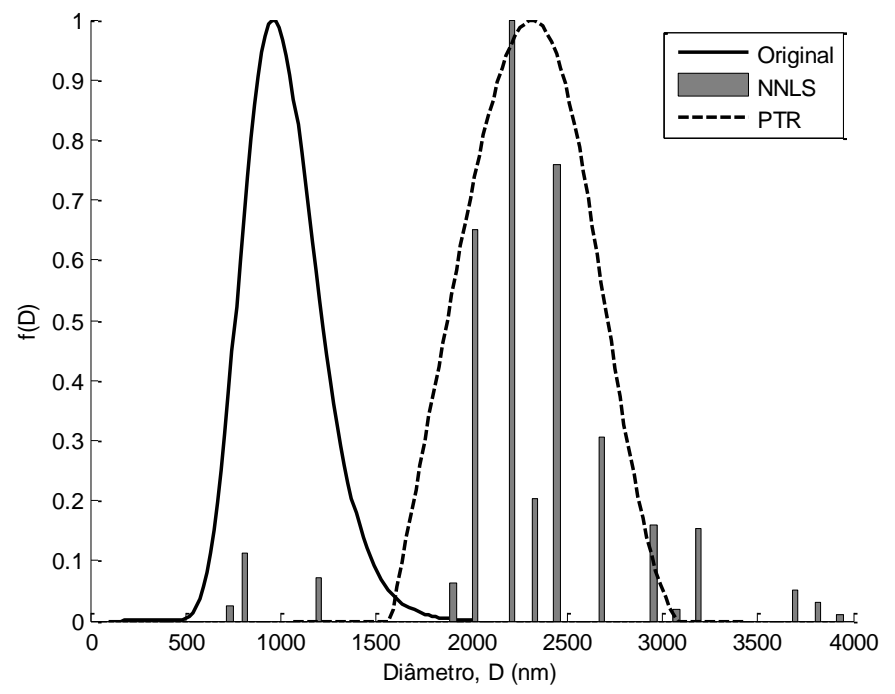

Figura 2 - Diâmetro médio $=1019,94$ nm e DP $=205,41$

As Figuras 1 e 2 mostram os resultados fornecidos pelas técnicas NNLS e PTR para diâmetros médios da distribuição original de até aproximadamente $1000 \mathrm{~nm}$. Nota-se que o posicionamento das distribuições recriadas pelos algoritmos estão deslocadas para diâmetros maiores que os originais. Os resultados fornecidos pelo algoritmo NNLS, no entanto, apresentaram uma maior dispersão, e o 
formato das distribuições estão mais próximos de uma curva bimodal do que uma monomodal, diferindo consideravelmente da distribuição original.

As curvas fornecidas pelo algoritmo PTR apresentam um comportamento mais próximo das distribuições originais. Nos dois casos, as curvas recriadas por esse algoritmo tem o aspecto de distribuições monomodais, necessitando de um ajuste para que a dispersão de classes de tamanho com frequência diferente de zero seja menor.

Abaixo é mostrada uma situação em que o diâmetro médio da distribuição original assume um valor maior, próximo dos $2000 \mathrm{~nm}$.

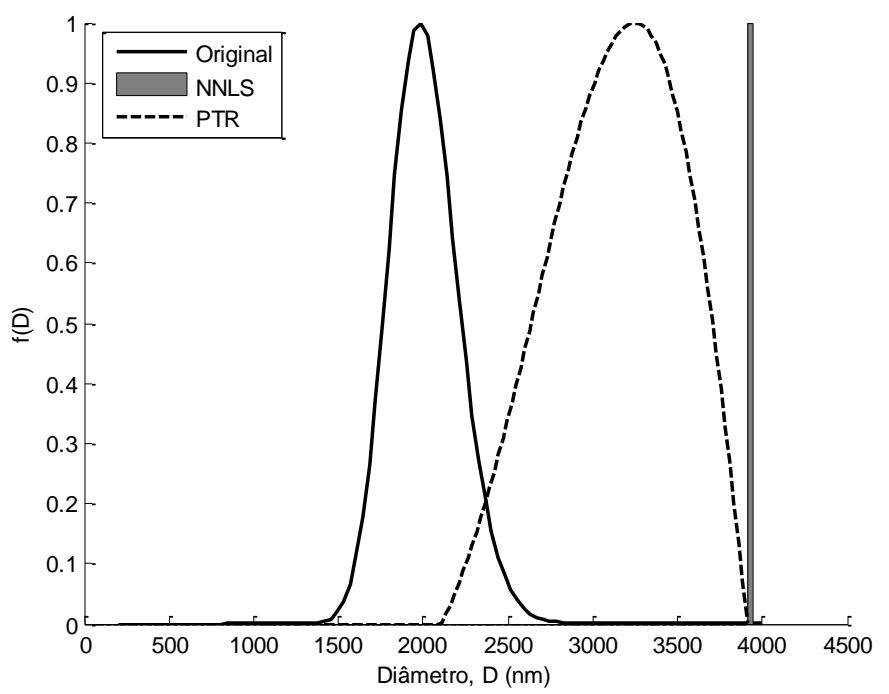

Figura 3 - Diâmetro médio = 2010,03 nm e DP = 201,51

A Figura 3 ressalta mais uma vez a diferença de qualidade nas distribuições fornecidas pelos algoritmos NNLS e PTR. A distribuição recriada pelo algoritmo NNLS apresentou apenas uma classe de tamanho com frequência diferente de zero, caracterizando uma distribuição monodispersa, muito distante da distribuição original. A distribuição recriada pelo algoritmo PTR, por sua vez, apresentou o aspecto de distribuição monomodal. Assim como nas Figuras 1 e 2, há a necessidade de ajustes para deslocar a distribuição para valores de tamanho de classe menores, além de diminuir a dispersão em torno da média, achatando a curva de distribuição.

O gráfico abaixo mostra o comparativo entre as distribuições originais artificiais e as distribuições recriadas a partir da técnica Non-Negative Least Squares. 


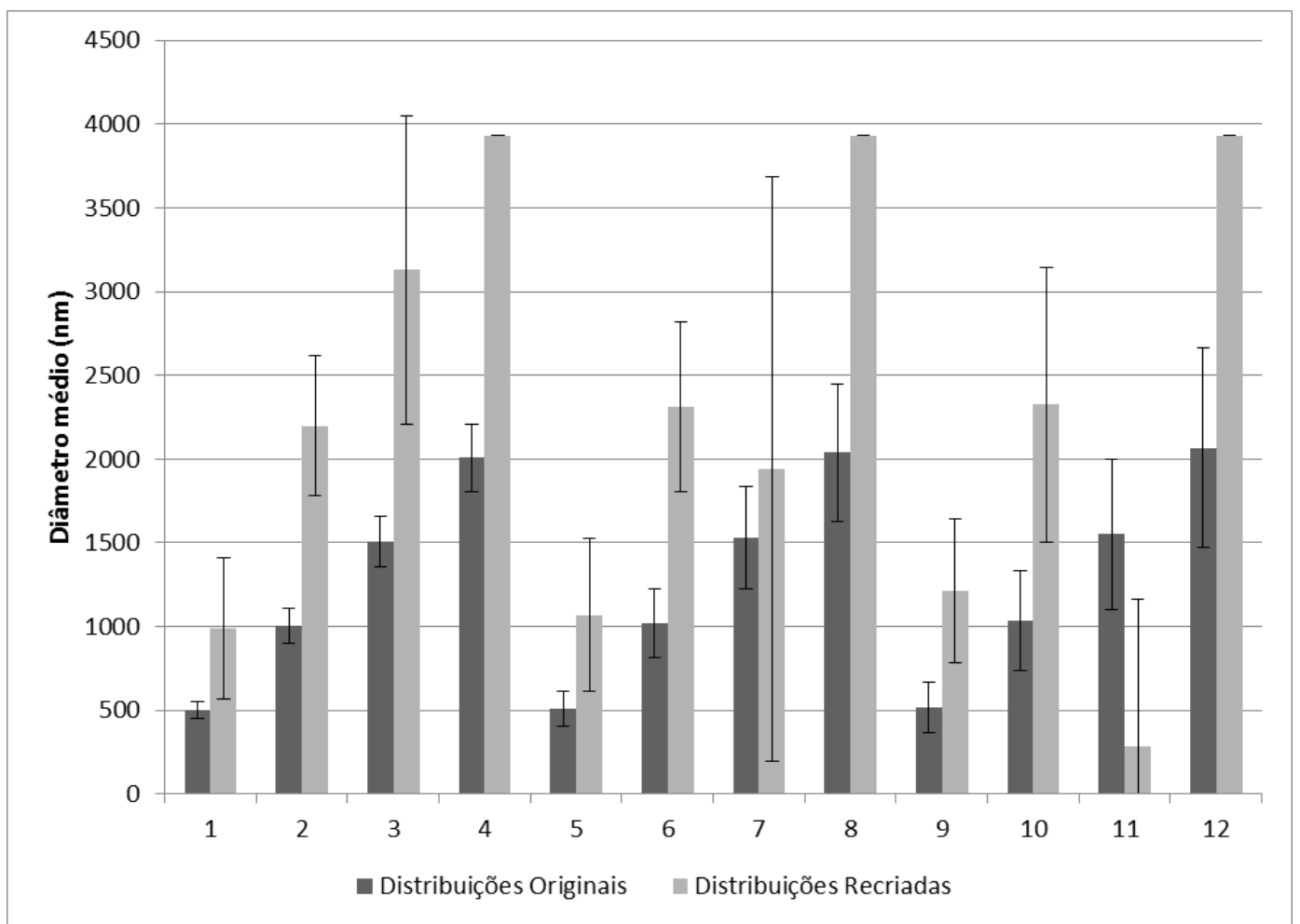

Figura 4 - Comparativo entre as distribuições originais e recriadas utilizando a técnica NNLS.

A Figura 4 ilustra a grande distância dos resultados fornecidos pelo algoritmo NNLS comparados às distribuições originais, que também pode ser visto nas Figuras 1, 2 e 3. Para os diâmetros maiores, as distribuições se comportam como na Figura 3, distribuição monodispersa, com dispersão nula em relação à média, o que justifica o desvio-padrão nulo nas amostras 4 , 8 e 12 na figura acima.

Para os diâmetros menores, o tamanho médio apresentou mais proximidade entre as distribuições originais e recriadas, que são os casos 1,5 e 9 na figura acima. No entanto, como é possível se ver na Figura 1, o formato das distribuições fornecidas por esse algoritmo assumem comportamento bimodal, muito diferente das distribuições originais, que apresentam aspecto monomodal.

A restrição de não-negatividade, apesar de forçar a não produção de resultados sem significado físico - frequências de tamanho de classe com valores negativos - implica em valores de resíduo maiores no processo de minimização comparados a outras técnicas convencionais, como por exemplo, a de mínimos quadrados sem restrições. O aumento no valor dos resíduos contribui para essas diferenças tão significativas entre as distribuições originais e recriadas.

A seguir, um gráfico que mostra um comparativo entre as distribuições originais e as distribuições recriadas através da técnica Philips-Twomey Regularization. 


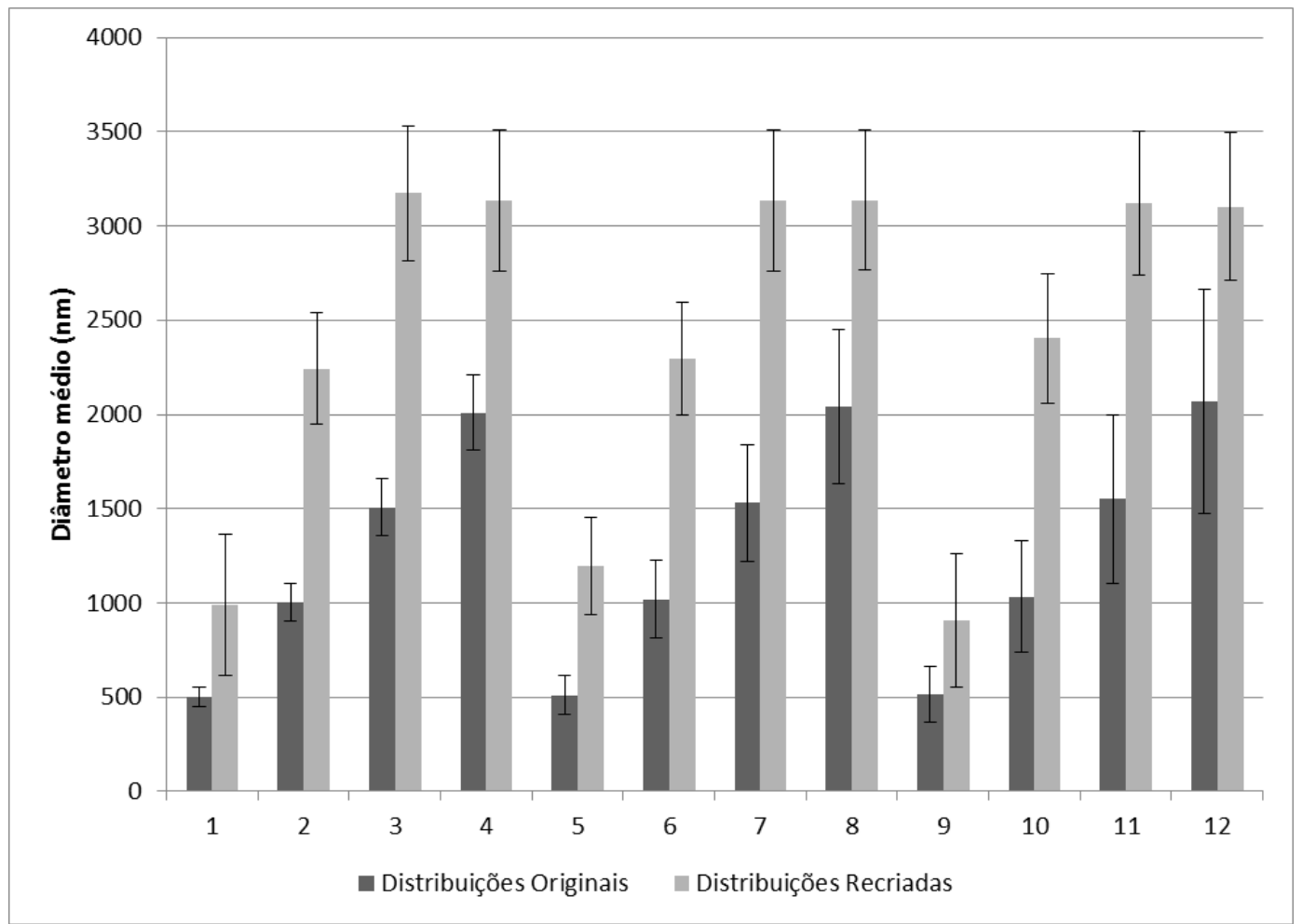

Figura 5 - Comparativo entre as distribuições originais e recriadas utilizando a técnica PTR.

Nota-se que as distribuições com diâmetros menores fornecidas pelo algoritmo PTR estão mais próximas das distribuições originais, comparando-se com os resultados fornecidos pelo algoritmo NNLS. Na figura acima, tem-se por exemplo, o caso 9 em que as médias podem ser consideradas estatisticamente iguais, e no caso 1, em que elas estão muito próximas. No entanto, para os diâmetros maiores, ocorre uma perda de sensibilidade no processo de recriação das distribuições. Para distribuições originais diferentes, as recriadas assumiram valores de média e desvio-padrão muito próximos e constantes. Isso evidencia que este algoritmo precisa de maiores ajustes nessas condições, ou que ainda, outros algoritmos sejam desenvolvidos para que se adequem a essa faixa de diâmetro.

É importante lembrar que a restrição de suavização existente nesse algoritmo contribui para a melhora da qualidade das distribuições fornecidas. Essa restrição evita que oscilações na curva resultante sejam evidenciadas, o que poderia produzir, por exemplo, distribuições bimodais ou de modas maiores. A ausência dessa restrição no algoritmo NNLS faz com que as oscilações nos valores de frequência adjacentes fiquem livres, o que resulta em distribuições com mais de uma moda e, consequentemente, menos próximas do resultado desejado.

\section{CONCLUSÃO}

A técnica PTR apresentou resultados mais consistentes comparados àqueles fornecidos pelo algoritmo NNLS. As distribuições obtidas pelo algoritmo PTR, para diâmetros de até $1000 \mathrm{~nm}$, tiveram comportamento semelhante (aspecto monomodal) ao das distribuições originais. Elas se 
apresentaram deslocadas para tamanhos de classe maiores e também mais dispersas que as distribuições originais, o que implica na necessidade de ajustes no algoritmo. No entanto, apresentaram maior sensibilidade do que para distribuições com tamanho médio de partículas grandes (acima de $1500 \mathrm{~nm}$ ).

A técnica NNLS, por sua vez, apresentou resultados pouco satisfatórios. Para valores de tamanho médio de partícula pequenos, a comparação de diâmetro médio entre as distribuições recriadas e originais ficaram próximas, porém as distribuições apresentaram forma bimodal. Para valores de diâmetro maiores, o algoritmo se mostrou pouco sensível aos espectros artificiais, resultando em distribuições monodispersas, resultado muito distante do desejado.

\section{AGRADECIMENTOS}

Os autores gostariam de agradecer à CAPES, CNPq e BRAGECRIM pelo apoio a esta pesquisa.

\section{REFERÊNCIAS}

BOHREN, C. F.; HUFFMAN, D. R. Absorption and Scattering of Light by Small Particles. New York: Editora John Wiley \& Sons, Inc., 1983.

ELICABE, G. E.; GARCIA-RUBIO, L. H. Latex particle size distribution from turbidimetry using a combination of regularization techniques and generalized cross validation. Advances in chemistry series, v. 227, p. 83-104, 1990.

LAWSON, C. L.; HANSON, R. J. Solving Least Squares Problems. Englewood Cliffs: Editora Prentice-Hall, 1974.

MÄTZLER, C. (2002a). MATLAB ${ }^{\circledR}$ Functions for Mie Scattering and Absorption. Bern, 2002.

MÄTZLER, C. (2002b). MATLAB ${ }^{\circledR}$ Functions for Mie Scattering and Absorption Version 2. Bern, 2002.

SEINFELD, J.; PANDIS, S. Atmospheric chemistry and physics: from air pollution to climate change. New Jersey: Editora John Wiley \& Sons, Inc., 2006.

TWOMEY, S. Introduction to the Mathematics of Inversion in Remote Sensing and Indirect Measurements. Mineola: Editora Elsevier Science Ltd., 1977. 